\title{
Review of SSA results on deuteron at COMPASS
}

\author{
A. Richter \\ on behalf of the COMPASS collaboration \\ Physikalisches Institut, Universität Erlangen-Nürnberg, \\ Erwin-Rommel-Str. 1, 91058 Erlangen, Germany \\ E-mail: andreas.richter@pi4.physik.uni-erlangen.de \\ www.pi4.physik.uni-erlangen.de
}

\begin{abstract}
The measuring of transverse single spin asymmetries (SSA) is part of the physics program at COMPASS, a fixed target experiment at CERN SPS. In 2002-04 the COMPASS experiment has collected data with a transversely polarised ${ }^{6} \mathrm{LiD}$ target using a $160 \mathrm{GeV} / \mathrm{c}$ polarised $\mu^{+}$beam. By measuring transverse SSA one has access to the transversity distribution function $\Delta_{T} q(x)$. This is one of the three quark distribution functions, which are needed to fully describe the spin structure of the nucleon at leading twist. At COMPASS three different quark polarimeters have been used to access transversity: the Collins effect, which produces an azimuthal asymmetry in the single hadron distribution, the azimuthal target spin asymmetries of charged hadron pairs and the transverse polarisation of $\Lambda$ hyperons.

In addition the SSA arising from the correlation between the transverse nucleon spin and the quark intrinsic transverse momentum (Sivers effect), was measured, together with six more transverse target spin asymmetries.

All the asymmetries measured on the deuteron target are small and compatible with zero.

Keywords: Transversity; Transverse Spin Asymmetry; Collins Asymmetry; Sivers Asymmetry; Interference Fragmentation Function; COMPASS.
\end{abstract}

\section{Introduction}

To fully describe the quark spin structure of the nucleon at leading twist three distribution functions are needed: The momentum distribution $q(x)$, which describes the probability of finding a quark with a fraction $x$ of the nucleon momentum, the helicity distribution $\Delta q(x)$, which describes the probability in a longitudinally polarised nucleon (w.r.t. the direction of motion) of finding a quark with spin parallel to the nucleon spin and the transversity distribution $\Delta_{T} q(x)$, which describes the probability in a transversely polarised nucleon (w.r.t. the direction of motion) of finding a 
quark with spin parallel to the nucleon spin. While the first two are known, transversity is now under intensive examination.

Since transversity is chiral-odd, it decouples from inclusive deep inelastic scattering (DIS). It is possible to measure it in semi-inclusive deep inelastic scattering (SIDIS) in combination with chiral-odd fragmentation functions, like the Collins fragmentation function $\Delta_{T}^{0} D_{q}^{h}$ for single hadron production or the interference fragmentation function $H_{1}^{\varangle}$ for the production of hadron pairs, which give rise to an azimuthal single spin asymmetry (SSA) in the final state hadrons.

A third channel, in which transversity was measured at COMPASS, is the transverse polarisation of $\Lambda$ hyperons, which is not part of this contribution A different mechanism, where SSA are created from the correlation between the nucleon spin and the quark intrinsic transverse momentum $k_{T}$ is the Sivers effect. ${ }^{1}$ It is described by the so-called Sivers distribution function $\Delta_{T}^{0} q\left(x, k_{T}\right)$.

In semi-inclusive DIS of polarised leptons on a transversely polarised target eight azimuthal modulations appear in the cross section. Within QCD parton model four azimuthal asymmetries can be interpreted at leading order, two of them being the already discussed Collins and Sivers asymmetries. The other two leading twist asymmetries, related to different transverse momentum dependent quark distribution functions, and also additional four asymmetries, which can be interpreted as twist-three contributions, have also been measured for the first time at COMPASS.

The COMPASS eperiment ${ }^{2}$ is a fixed target experiment, which uses a polarised $\mu^{+}$beam with a momentum of $160 \mathrm{GeV} / \mathrm{c}$ at the CERN SPS M2 beamline. The main goal of its spin physics program is the study of the spin structure of the nucleon. In 2002-04 a polarised ${ }^{6} \mathrm{LiD}$ target was used, whereas the target nucleons had a polarisation transverse to the beam direction for nearly $20 \%$ of the beam time in this years.

\section{One hadron asymmetries}

\subsection{Collins asymmetry}

The Collins mechanism ${ }^{3}$ leads to a modulation in the azimuthal distribution of the inclusively produced hadrons given by:

$$
N=N_{0} \cdot\left(1+f \cdot P_{T} \cdot D_{N N} \cdot A_{C} \cdot \sin \left(\Phi_{C o l l}\right)\right)
$$

where $P_{T}$ is the target polarisation $(\sim 50 \%), f$ the target dilution factor $(\sim 0.38)$, i.e. the fraction of the target nuclei, which can be polarised, 
$D_{N N}=(1-y) /\left(1-y+y^{2} / 2\right)$ the spin transfer coefficient. $\Phi_{C o l l}$ is the Collins angle, defined as $\Phi_{h}-\Phi_{S^{\prime}}=\Phi_{h}+\Phi_{S}-\pi$, where $\Phi_{h}$ is the azimuthal angle of the hadron momentum, $\Phi_{S^{\prime}}$ is the azimuthal angle of the quark spin after scattering and $\Phi_{S}$ is the azimuthal angle of the nucleon spin in the $\gamma-N$ system.

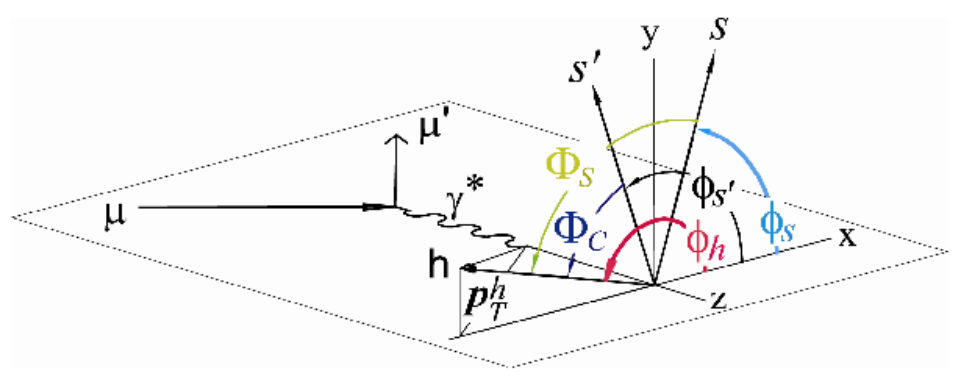

Fig. 1. Definition of Collins and Sivers angle.

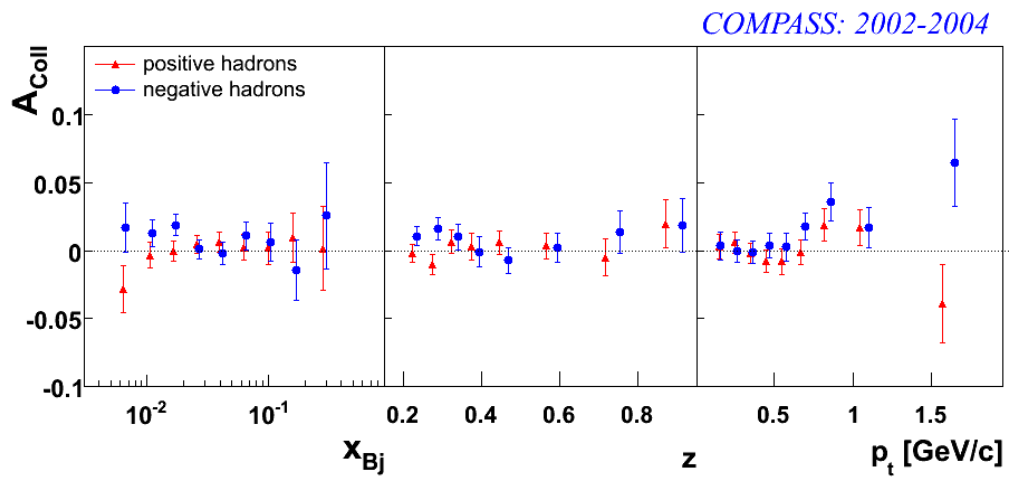

Fig. 2. Collins asymmetries for unidentified hadrons as a function of $x_{b j}, z$ and $p_{t}$.

$A_{C}$ is the Collins asymmetry and is given by:

$$
A_{C}=\frac{\sum_{q} e_{q}^{2} \Delta_{T} q(x) \Delta_{T}^{0} D_{q}^{h}\left(z, p_{T}^{h}\right)}{\sum_{q} e_{q}^{2} q(x) D_{q}^{h}\left(z, p_{T}^{h}\right)}
$$


The Collins asymmetry is proportional to the convolution of the transversity distribution $\Delta_{T} q(x)$ and the Collins fragmentation function $\Delta_{T}^{0} D_{q}^{h}$ divided by the momentum distribution $q$ and the unpolarised fragmentation function $D_{q}^{h} \cdot z=E_{h} /\left(E_{l}-E_{l^{\prime}}\right)$ is the fraction of the virtual photon energy carried by the hadron and $p_{T}^{h}$ is the transverse momentum of the hadron with respect to the direction of the virtual photon.

The event selection requires standard DIS cuts: photon virtuality $Q^{2}>$ $1(\mathrm{GeV} / \mathrm{c})^{2}, 0.1<y<0.9$ and mass of the final hadronic state $W>$ $5 \mathrm{GeV} / \mathrm{c}^{2}$. Additional cuts on the detected hadrons are: $z>0.2$ and $p_{T}^{h}>0.1$.

The Collins asymmetry of unidentified hadrons ${ }^{4,5}$ is shown in fig. 2 as a function of $x_{b j}, z$ and $p_{T}^{h}$; the asymmetries are small and compatible with zero. Only the statistical error is shown in the error bars, while several studies have shown that the systematic errors are much smaller than the statistical ones. The most energetic (leading) hadrons show a similar behavior to that of all detected hadrons.

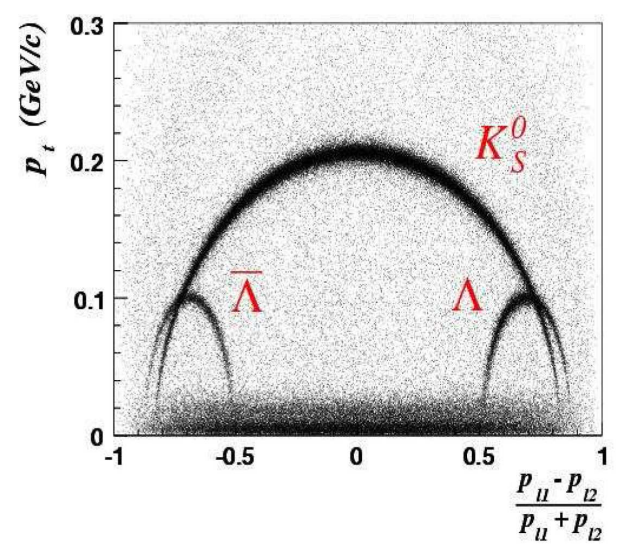

Fig. 3. Armenteros plot of the hadron pair.

The same analysis done for the charged hadrons has been repeated to extract the Collins and Sivers asymmetry for $\pi^{ \pm}$and $K^{ \pm}$for the data collected in 2003 and 2004, using the information of the Ring Imaging Cherenkov Detector detector (RICH) to identify the hadrons, and for $K_{S}^{0}$ for the whole data from 2002-04. The COMPASS RICH1 detector uses $\mathrm{C}_{4} \mathrm{~F}_{10}$ as radiator gas, with a refractive index of $\sim 1.0015$ for $7 \mathrm{eV}$ Cherenkov photons, that 
gives a threshold of 2 (9) $\mathrm{GeV}$ for pions (kaons) and allows to separate pions from kaons up to $45-50 \mathrm{GeV}$. More than $80 \%$ of the hadrons in this energy interval identified by the RICH are pions and the rest are kaons.

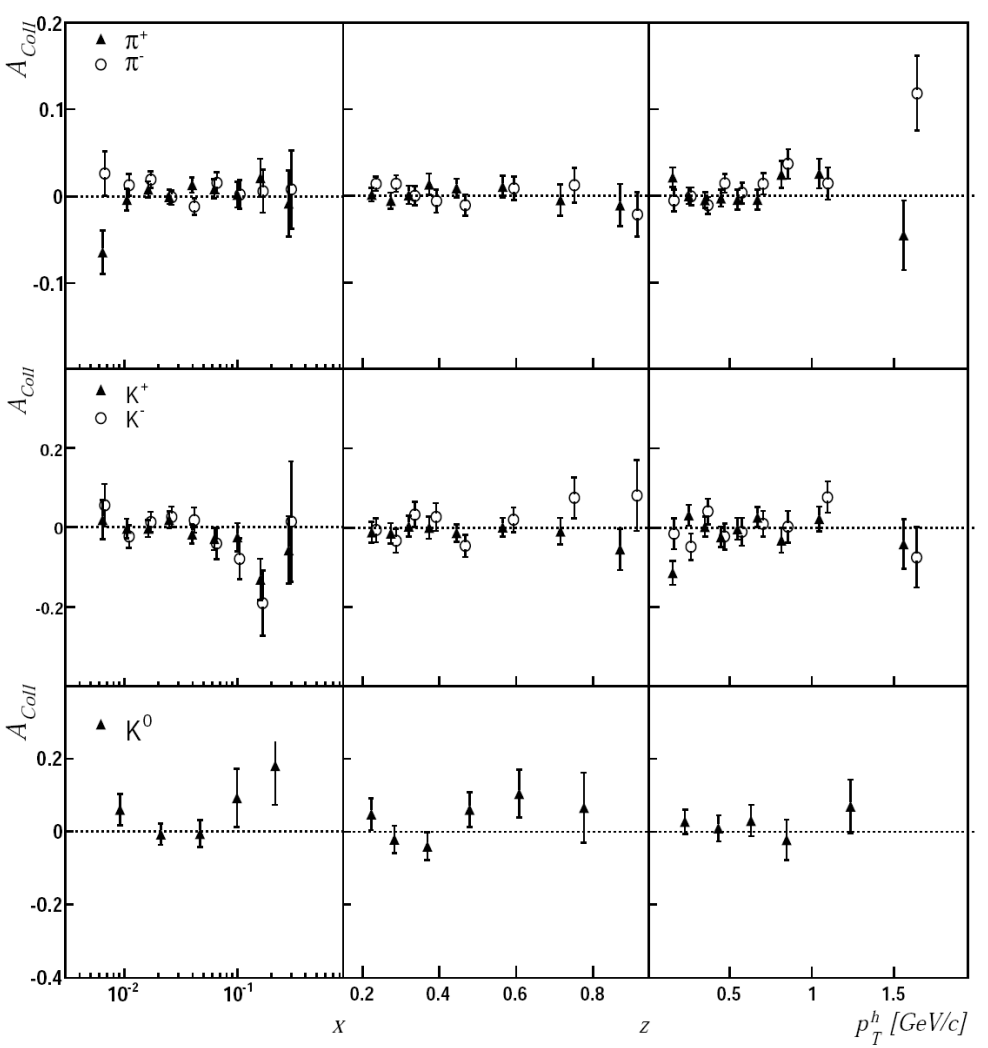

Fig. 4. Collins asymmetries for identified hadrons (pions, kaons) as a function of $x_{b j}$, $z$ and $p_{t}$.

$K_{S}^{0}$ have been identified by using secondary vertices with two outgoing and no incoming particle and calculating the invariant mass of the decay products assumed to be pions. Further cuts are on the transverse momentum of one of the hadrons relative to the hadron momentum sum $\left(p_{t}>0.025 \mathrm{GeV} / \mathrm{c}\right.$, see Armenteros plot: fig. 3), on the primary vertex pointing of the $K_{S}^{0}$ and on a minimum distance between the primary vertex and the forward secondary vertex at least $10 \mathrm{~cm}$. With this cuts a signal 
to background ratio of 15 for the $K_{S}^{0}$ sample is reached.

The Collins asymmetry of the identified hadrons ${ }^{6}$ are shown in fig. 4. Like for the unidentified hadrons, the asymmetries are small and compatible with zero.

Although there were some predictions for small asymmetries by assuming an opposite contribution from $u$ and $d$ quarks, causing a large cancellation for the isoscalar deuteron target at COMPASS regimes, there was no clear expectation that the asymmetries are so small. On the ( $u$-dominated) proton the HERMES collaboration has measured a non-zero Collins asymmetry, ${ }^{7}$ leading to the conclusion that both, the transversity distribution $\Delta_{T} u(x)$ and the Collins fragmentation function $\Delta_{T}^{0} D_{u}^{h}$, are non-zero.

As the Collins fragmentation function is measured at the Belle experiment, ${ }^{8}$ it is possible to do an extraction of the transversity distributions. ${ }^{9}$ Hereby the COMPASS deuteron data allow, in conjunction to the HERMES data on proton and the Belle data in $e^{+} e^{-}$hadron production, to extract $\Delta_{T} d(x)$.

\subsection{Sivers asymmetry}

The Sivers asymmetry gives a modulation in the azimuthal distribution of $\Phi_{\text {Siv }}=\Phi_{h}-\Phi_{S}$, which is independent of the Collins angle and can therefore be disentangled in SIDIS.

COMPASS has published results for the unidentified ${ }^{4,5}$ as well as for the identified hadrons $\left(\pi^{ \pm}, K^{ \pm}, K_{S}^{0}\right){ }^{6}$ The asymmetries are small and compatible with zero (see fig. 5). Given the non-zero asymmetry measured by HERMES for Sivers, also there might be a cancellation between $u$ and $d$, i.e. $\Delta_{T}^{0} d \sim-\Delta_{T}^{0} u$. For a summary concerning a simultaneous description of the COMPASS and HERMES data see: ${ }^{10}$ Furthermore the vanishing of the Sivers asymmetry on deuteron can be interpreted as the absence of gluon orbital angular momentum in the nucleon. ${ }^{11}$

\subsection{Other single spin asymmetries}

In the SIDIS cross-section there are alltogether eight structure functions, which depend on the transverse target polarisation and cause eight spin dependent asymmetries with different, independent azimuthal modulations. All of them were being measured at COMPASS. Two of them are the Collins and the Sivers asymmetry. Another two are also twist-2 asymmetries and can therefore be interpreted in the QCD parton model. They will allow to extract two further quark distribution functions. The remaining six can be 


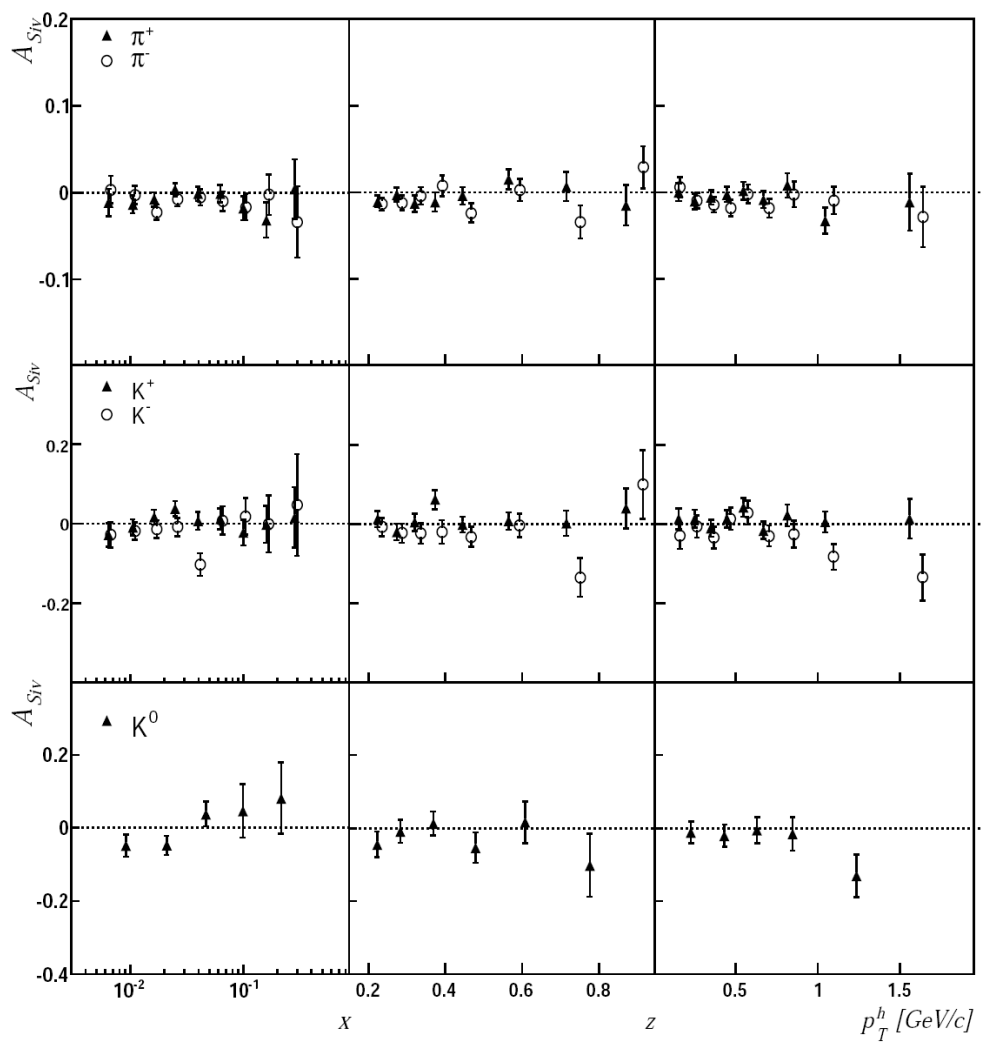

Fig. 5. Sivers asymmetries for identified hadrons (pions, kaons) as a function of $x_{b j}, z$ and $p_{t}$.

interpreted as twist-3 contributions. All those asymmetries are found to be very small and compatible with zero.

\section{Two hadron asymmetries}

$\Delta_{T} q(x)$ can also be accessed via the production of pairs of two unpolarised hadrons. ${ }^{12,13}$ The two hadron production counting rates are given by:

$$
N=N_{0} \cdot\left(1+f \cdot P_{T} \cdot D_{N N} \cdot A_{R S} \cdot \sin \left(\Phi_{R S}\right)\right)
$$

The azimuthal angle $\Phi_{R S}$ is defined as $\Phi_{R S}=\Phi_{R}-\Phi_{S}-\pi$, with $\Phi_{R}$ the azimuthal angle of the plane containing the two hadrons and $\Phi_{S}$ the azimuthal angle of the target spin vector with respect to the lepton scattering 
plane. $\Phi_{R}$ is defined at COMPASS as the azimuthal angle of the transverse component $R_{T}$ of the vector $\frac{z_{2} \mathbf{P}_{1}-z_{1} \mathbf{P}_{2}}{z_{1}+z_{2}}$. Hereby the indices 1 and 2 refer to the two final state hadrons.

The asymmetry $A_{R S}$ is given by:

$$
A_{R S}=\frac{\sum_{q} e_{q}^{2} \Delta_{T} q(x) H_{1}^{\varangle}\left(z, M_{h}^{2}\right)}{\sum_{q} e_{q}^{2} q(x) D_{q}^{h}\left(z, M_{h}^{2}\right)}
$$

$M_{h}$ is here the invariant mass of the hadron pair. $D_{q}^{h}\left(z, M_{h}^{2}\right)$ is the also unknown unpolarised fragmentation function into two hadrons. The asymmetry is proportional to the convolution of the transversity distribution $\Delta_{T} q(x)$ and the interference fragmentation function $H_{1}^{\varangle}\left(z, M_{h}^{2}\right)$ that can be measured at the Belle experiment.

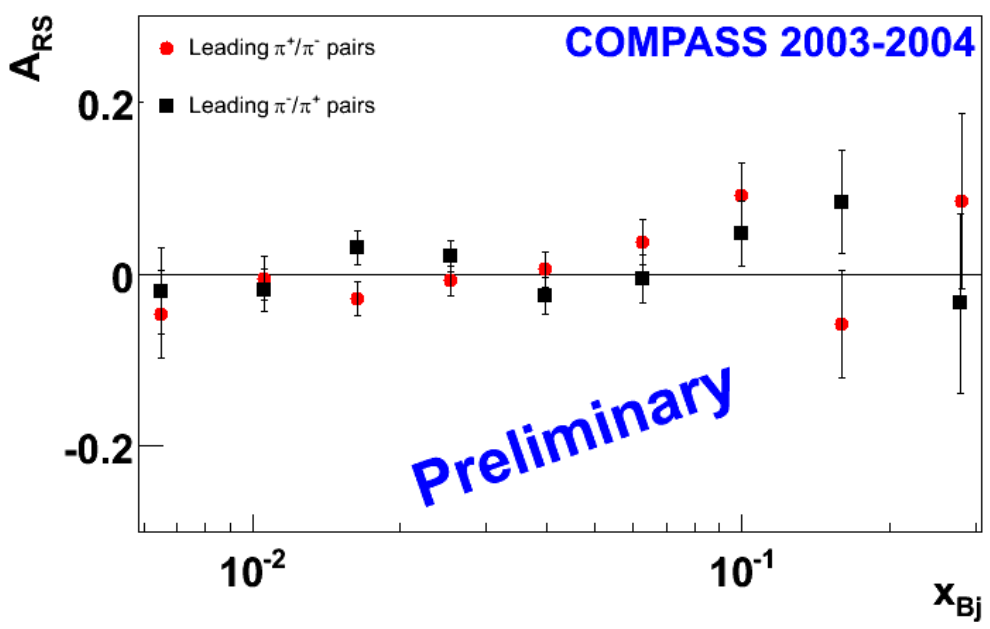

Fig. 6. Asymmetries of leading $\pi^{+} \pi^{-}$hadron pairs as a function of $x_{b j}$.

There were two different analysis of the data: first all hadron pairs with a positive and a negative hadron were considered, giving four combinations for identified hadrons: $\pi^{+} \pi^{-}, \pi^{+} K^{-}, K^{+} \pi^{-}$and $K^{+} K^{-}$.

Secondly, a $z$ ordered data sample was selected, using only the leading and the sub-leading hadron (in $z$ ) of the event. Here also combinations of hadrons with the same charge are possible. 
The event selection is basically the same as for the single hadron asymmetries, with the exception of the $z$-cut, which was $z_{1,2}>0.1$ for each hadron and $z_{1}+z_{2}<0.9$ for the sum to remove exclusive production.

The asymmetries were studied as function of $x_{b j}, z$ and $M_{h}$. No clear signal variant from zero was seen (for one example see fig. 6). For its proton target the HERMES collaboration has measured non-zero results. ${ }^{14}$

\section{Summary}

In 2002-04 the COMPASS collaboration has measured a full set of single spin asymmetries on a transversely polarised deuterium target. The asymmetries on the deuteron were all found to be small and compatible with zero, which implicates a cancellation of the $u$ and $d$ quark contribution to the asymmetries in a deuterium target.

In 2007 COMPASS has also measured transverse asymmetries on a polarised proton target. ${ }^{15}$

\section{References}

1. D. W. Sivers, Phys. Rev. D 41, 83 (1990).

2. P. Abbon et al., [COMPASS collaboration] Nucl. Instrum. Meth. A 577, 455 (2007).

3. J. C. Collins, Nucl. Phys. B 396, 161 (1993).

4. V. Y. Aleaxakhin et al., [COMPASS collaboration] Phys. Rev. Lett. 94, 202002 (2005).

5. E. S. Ageev et al., [COMPASS collaboration] Nucl. Phys. B 765, 31 (2007).

6. M. Alexeev et al., [COMPASS collaboration]; arXiv:0802.2160 [hep-ex] (2008).

7. M. Diefenthaler et al., [HERMES collaboration] proceedings of DIS 2007, 579 (2007); arXiv:0706.2242 [hep-ex].

8. K. Abe et al., [Belle collaboration] Phys. Rev. Lett. 96, 232002 (2006).

9. M. Anselmino et al.; arXiv:0701006v3 [hep-ph] (2007).

10. M. Anselmino et al., proceedings of Transversity 2005; arXiv:0511017v1 [hepph] (2005).

11. S. J. Brodsky, S. Gardner, Phys. Lett. B 643, 22 (2006).

12. A. Bacchetta, M. Radici, proceedings of DIS 2004; arXiv:0407345 [hep-ph] (2004).

13. X. Artru, proceedings of 10th Rhodanien Seminar: The Spin in Physics; arXiv:0207309 [hep-ph] (2002).

14. A. Airapetian et al., [HERMES collaboration]; arXiv:0803.2367v2 [hep-ex] (2008).

15. S. Levorato, [COMPASS collaboration], these proceedings. 